\title{
Trace distance from the viewpoint of quantum operation techniques
}

\author{
Alexey E. Rastegin \\ Department of Theoretical Physics, Irkutsk State University, Gagarin Bv. 20, \\ Irkutsk 664003, Russia
}

\begin{abstract}
In the present paper, the trace distance is exposed within the quantum operations formalism. The definition of the trace distance in terms of a maximum over all quantum operations is given. It is shown that for any pair of different states, there are an uncountably infinite number of maximizing quantum operations. Conversely, for any operation of the described type, there are an uncountably infinite number of those pairs of states that the maximum is reached by the operation. A behaviour of the trace distance under considered operations is studied. Relations and distinctions between the trace distance and the sine distance are discussed.
\end{abstract}

03.67.-a, 03.65.Ta, 02.10.Yn

\section{Introduction}

The formalism of quantum operations provides a unified treatment of possible state change in quantum theory [1, 2]. The key results on the subject of quantum operations have their origins in papers by Hellwig and Kraus [3, 4], by Kraus [5], by Lindblad [6] and by Choi [7]. The two basic transformations, the unitary evolution and the projective measurement, are the simplest examples of quantum operations. But very different operations are just needed in quantum information processing. For example, we consider distinguishing two non-orthogonal states. This task arises in the quantum cryptography protocol B92 [8] and binary optical communication [9]. The 
well-known scheme proposed by Helstrom [10] is not error-free (except the case of orthogonality). Nevertheless, if we allow inconclusive answers then probabilistic error-free distinction is possible [11, 12, 13]. This scheme is usually referred to as unambiguous discrimination [14, 15, 16]. The nonorthogonality of states to be distinguished means that no projective measurement can hit. Here we must look to generalized measurements [13, 17]. As it is shown in [14, 15], a rigorous treatment of arbitrary number of those signals that should be discriminated is naturally dealt within the quantum operations techniques.

In the light of those topics that are the subject of active research, the techniques of quantum operations gain significance of standard powerful tool. Indeed, many important protocols can be recast as special cases of quantum operation; for instance, the broadcasting [18], the teleportation [19], the state separation [20, 21, 22], and the procedure that interpolates between unambiguous discrimination and the Helstrom scheme [23]. A model of computations with mixed states is formally posed in terms of trace-preserving quantum operations [24]. So, it is utmost importance that we should have an operational meaning of basic notions of quantum theory. The revision of needed background within the quantum operations formalism may provide a new viewpoint on the habitual concepts. The aim of the present work is to give a combined exposition of the trace distance and the quantum operations in one location. We will also discuss concerning questions.

The paper is organized as follows. In the remainder of this section, we briefly recall necessary tools of quantum operations techniques. In Section 2 we offer a non-standard definition of the trace distance. Due to this new definition, a certain subclass of quantum operations will be specified. Each of these operations maximizes a difference between two probabilities that are generated by the operation on some pairs of inputs. For given pair of inputs, there is uncountably set of such maximizing operations. On other hand, for any quantum operation of specified type, there is uncountably set of input pairs with described property. In Section 3 a change of the trace distance under the maximizing operation is examined. If an operation maximizes difference between probabilities generated by inputs then the trace distance between outputs is bounded above. We also discuss statistical properties of this change of the trace distance. In Section 4 relations of the trace distance to the sine distance are considered. The bounds on the maximum of difference between these distances are given. Section 5 concludes the paper with a summary of obtained results. 
Let $\mathcal{H}_{1}$ and $\mathcal{H}_{2}$ be the finite-dimensional Hilbert spaces. In general, these spaces are assumed to be different. To mark distinction of spaces, we shall supply the item of trace operation by a label. That is, the $\operatorname{trace} \operatorname{tr}_{1}\{\cdot\}$ is taken over $\mathcal{H}_{1}$, the trace $\operatorname{tr}_{2}\{\cdot\}$ is taken over $\mathcal{H}_{2}$. Consider any process $\mathcal{E}$ that leads to a map

$$
\rho \rightarrow \rho^{\prime}:=\frac{\mathcal{E}(\rho)}{\operatorname{tr}_{2}\{\mathcal{E}(\rho)\}},
$$

where an input $\rho$ is some normalized state on $\mathcal{H}_{1}$ and an output $\rho^{\prime}$ is some normalized state on $\mathcal{H}_{2}$. If this map is consistent with the laws of quantum theory, then $\mathcal{E}$ is a quantum operation with the input space $\mathcal{H}_{1}$ and the output space $\mathcal{H}_{2}[2]$. The normalizing divisor in (1.1) is the probability that the above process occurs. So we demand that

$$
0 \leq \operatorname{tr}_{2}\{\mathcal{E}(\rho)\} \leq 1
$$

for each input $\rho$. In addition, a map $\mathcal{E}$ must be linear and completely positive [2].

The operator-sum representation is a key result of the quantum operations formalism. Namely [1, 2], the map $\mathcal{E}$ is a quantum operation if and only if

$$
\mathcal{E}(\rho)=\sum_{\mu} \mathrm{E}_{\mu} \rho \mathrm{E}_{\mu}^{\dagger}
$$

for some set of operators $\left\{\mathrm{E}_{\mu}\right\}$. These operators map the input space $\mathcal{H}_{1}$ to the output space $\mathcal{H}_{2}$. Some features of given quantum operation are determined by properties of the positive operator

$$
\mathrm{T}:=\sum_{\mu} \mathrm{E}_{\mu}^{\dagger} \mathrm{E}_{\mu} .
$$

In the following, we will essentially use the equality

$$
\operatorname{tr}_{2}\{\mathcal{E}(\rho)\}=\operatorname{tr}_{1}\{\mathbf{T} \rho\} .
$$

This is based on the operator-sum representation and the properties of the trace. Suppose $\mathrm{A}: \mathcal{H}_{1} \rightarrow \mathcal{H}_{2}$ and $\mathrm{B}: \mathcal{H}_{2} \rightarrow \mathcal{H}_{1}$ are linear operators. Then by the cyclic property we have $\operatorname{tr}_{2}\{A B\}=\operatorname{tr}_{1}\{B A\}$. Tracing the right-hand side of (1.3) and using the cyclic property and the linearity of the trace, we at once obtain (1.5). The inequality (1.2) must be satisfied for all inputs. Combining this with (1.5), we get $\mathbf{0} \leq \mathbf{T} \leq \mathbf{1}$. 
We shall also use the fact [2] that operator $(\rho-\varrho)$ can be represented as $\rho-\varrho=\mathbf{Q}-\mathbf{R}$, where $\mathbf{Q}$ and $\mathbf{R}$ are positive operators with the orthogonal support spaces. [Recall that support of an operator is defined as the vector space orthogonal to its kernel.] Indeed, due to the spectral decomposition of $(\rho-\varrho)$ we obtain

$$
\begin{aligned}
& \mathbf{Q}:=\sum_{q} \lambda_{q}|q\rangle\langle q|, \\
& \mathbf{R}:=\sum_{r} \varkappa_{r}|r\rangle\langle r|,
\end{aligned}
$$

where the $\lambda_{q}$ 's and the $\left(-\varkappa_{r}\right)$ 's are strictly positive and strictly negative eigenvalues of operator $(\rho-\varrho)$ respectively. Let $\operatorname{supp}(\mathrm{A})$ denote the support of an operator $\mathrm{A}$. Then the input space $\mathcal{H}_{1}$ can be expressed as

$$
\mathcal{H}_{1}=\operatorname{supp}(\mathbf{Q}) \oplus \operatorname{supp}(\mathbf{R}) \oplus \mathcal{K},
$$

where $\operatorname{supp}(\mathbf{Q})$ is spanned by $|q\rangle$ 's, $\operatorname{supp}(\mathbf{R})$ is spanned by $|r\rangle$ 's and $\mathcal{K}$ denotes the kernel of operator $(\rho-\varrho)$.

\section{Non-standard definition}

In this section, we shall introduce a non-standard definition of trace distance and investigate those questions that are risen in the planned way. With each quantum operation $\mathcal{E}$, one can associate some distance measure for quantum states. Let $\rho$ and $\varrho$ be the normalized states on $\mathcal{H}_{1}$. Two positive numbers $\operatorname{tr}_{2}\{\mathcal{E}(\rho)\}$ and $\operatorname{tr}_{2}\{\mathcal{E}(\varrho)\}$ give the probabilities that the represented process occurs when $\rho$ and $\varrho$ were respectively taken as initial state. It is natural to measure a closeness of these states by the difference between the corresponding probabilities.

Definition 1. Let $\mathcal{E}$ be a quantum operation. The $\mathcal{E}$-distance $d_{\mathcal{E}}(\rho, \varrho)$ between normalized states $\rho$ and $\varrho$ is defined by

$$
d_{\mathcal{E}}(\rho, \varrho):=\left|\operatorname{tr}_{2}\{\mathcal{E}(\rho)\}-\operatorname{tr}_{2}\{\mathcal{E}(\varrho)\}\right| .
$$

It is clear that $0 \leq d_{\mathcal{E}} \leq 1$, that if $\rho=\varrho$ then $d_{\mathcal{E}}(\rho, \varrho)=0$, and that $d_{\mathcal{E}}$ is a symmetric function of inputs. The absolute value of sum does not exceed the sum of absolute values so that $d_{\mathcal{E}}(\rho, \varrho) \leq d_{\mathcal{E}}(\rho, \omega)+d_{\mathcal{E}}(\omega, \varrho)$, i.e. the triangle inequality holds. So $\mathcal{E}$-distance obeys all the properties of a metric except only one. Namely, even if $\rho \neq \varrho$ the equality $d_{\mathcal{E}}(\rho, \varrho)=0$ can 
still be valid (when $\operatorname{dim}\left(\mathcal{H}_{1}\right)>2$ ). Indeed, due to (1.5) the last equality is equivalent to $\operatorname{tr}_{1}\{\mathbf{T}(\rho-\varrho)\}=0$ that is provided by $\operatorname{supp}(\mathbf{T}) \subseteq \mathcal{K}$. [Only in two-dimensional input space, $\mathcal{E}$-distance is a metric because $\rho \neq \varrho$ implies here that $\operatorname{dim}(\mathcal{K})=0$ is inevitable.] It is unfit that $d_{\mathcal{E}}(\rho, \varrho)=0$ does not imply $\rho=\varrho$. But this lack is repaired by the maximization over all quantum operations. It turns out that such an approach leads to well-known metric on quantum states, namely to the trace distance.

Let $|A|$ denote the positive square root of $A^{\dagger} A$ (for any positive operator there exists a unique positive square root [25]). The trace distance between states $\rho$ and $\varrho$ is traditionally defined by [2]

$$
D(\rho, \varrho):=\frac{1}{2} \operatorname{tr}_{1}|\rho-\varrho| .
$$

The trace distance is simply expressed in terms of operators $\mathbf{Q}$ and $\mathbf{R}[2]$. Since the supports of these operators are orthogonal, we have $|\mathbf{Q}-\mathbf{R}|=\mathbf{Q}+\mathbf{R}$ and

$$
D(\rho, \varrho)=\frac{1}{2} \operatorname{tr}_{1}(\mathbf{Q})+\frac{1}{2} \operatorname{tr}_{1}(\mathbf{R}) .
$$

When states $\rho$ and $\varrho$ are normalized to the unit trace, the right-hand side of (2.3) is equal to $\operatorname{tr}_{1}(\mathbf{Q})=\operatorname{tr}_{1}(\mathbf{R})$. The trace distance has many attractive properties that makes it a proper measure of closeness of quantum states (for a discussion, see subsection 9.2.1 of reference [2]). The mentioned connection between the $\mathcal{E}$-distance and the trace distance is established by the following statement.

Theorem 1. For any normalized states $\rho$ and $\varrho$,

$$
\max _{\mathcal{E}} d_{\mathcal{E}}(\rho, \varrho)=D(\rho, \varrho)
$$

where maximum is taken over all quantum operations $\mathcal{E}$. The maximum is reached by quantum operation $\mathcal{E}$ if and only if operator $\mathbf{T}$ is equal to either the projector onto $\operatorname{supp}(\mathbf{Q})$ or the projector onto $\operatorname{supp}(\mathbf{R})$, up to additive term $\mathbf{M}$ satisfying $\operatorname{supp}(\mathbf{M}) \subseteq \mathcal{K}$ and $\mathbf{0} \leq \mathbf{M} \leq \mathbf{1}$.

Proof. We shall now suppose that $\rho \neq \varrho$ (otherwise both distances are zero, $\mathcal{K}=\mathcal{H}_{1}$ and the statement of theorem does not add anything new). Then both sets $\left\{\lambda_{q}\right\}$ and $\left\{\varkappa_{r}\right\}$ are nonempty. Due to (1.5) we have

$$
d_{\mathcal{E}}(\rho, \varrho)=\left|\operatorname{tr}_{1}\{\mathbf{T Q}\}-\operatorname{tr}_{1}\{\mathbf{T R}\}\right| .
$$

Since operators $\mathbf{Q}$ and $\mathbf{R}$ are positive and $\mathbf{0} \leq \mathbf{T} \leq \mathbf{1}$, each of two traces in the right-hand side of (2.5) is nonnegative and no greater than $D(\rho, \varrho)=$ 
$\operatorname{tr}_{1}(\mathbf{Q})=\operatorname{tr}_{1}(\mathbf{R})$. So $\mathcal{E}$-distance between states $\rho$ and $\varrho$ does not exceed the trace distance between them. The equality is reached in two cases: (i) $\operatorname{tr}_{1}\{\mathbf{T Q}\}=\operatorname{tr}_{1}(\mathbf{Q})$ and $\operatorname{tr}_{1}\{\mathbf{T R}\}=0 ;\left(\right.$ ii) $\operatorname{tr}_{1}\{\mathbf{T Q}\}=0$ and $\operatorname{tr}_{1}\{\mathbf{T R}\}=$ $\operatorname{tr}_{1}(\mathbf{R})$. We shall consider the case (i) only; the case (ii) follows the same pattern. If $\mathbf{T}$ is the sum of projector onto $\operatorname{supp}(\mathbf{Q})$ and some $\mathbf{M}$ with $\operatorname{supp}(\mathbf{M}) \subseteq \mathcal{K}$ then the conditions of the case (i) take place. Suppose now that the conditions of the case (i) are fulfilled. Let the $|a\rangle$ 's form an orthonormal set in $\operatorname{supp}(\mathbf{Q}) \oplus \mathcal{K}$. Clearly, $\langle a \mid r\rangle=0$ for all $a$ and $r$. Then operator $\mathbf{T}$ can be expressed by

$$
\mathbf{T}=\sum_{a} c_{a a}|a\rangle\left\langle a\left|+\sum_{a r}\left(c_{a r}|a\rangle\left\langle r\left|+c_{r a}\right| r\right\rangle\langle a|\right)+\sum_{r} c_{r r}\right| r\right\rangle\langle r|,
$$

where all the diagonal elements lie in the interval $[0 ; 1]$. Because the $\varkappa_{r}$ 's in (1.6) are strictly positive, the condition $\operatorname{tr}_{1}\{\mathbf{T R}\}=0$ implies that $c_{r r}=0$ for all values of label $r$ (so the kernel of $\mathbf{T}$ is not zero-dimensional). Moreover, all the off-diagonal elements $c_{a r}$ and $c_{r a}$ are also zero. To prove this fact, we use a modification of the method of reference [26]. Let us fix the values of $a$ and $r$, and let us consider a subspace $\operatorname{span}\{|a\rangle,|r\rangle\}$. In this subspace, the action of $\mathbf{T}$ is described by the matrix

$$
\left(\begin{array}{cc}
c_{a a} & \alpha-i \beta \\
\alpha+i \beta & 0
\end{array}\right) .
$$

Here $\alpha$ and $\beta$ are real, and $c_{a r}^{*}=c_{r a}=\alpha+i \beta$. Due to positivity of $\mathbf{T}$, both eigenvalues of the matrix (2.7) are nonnegative. This is valid if and only if $\alpha=\beta=0$ and therefore $c_{a r}=c_{r a}=0$. Thus, only the first sum in the right-hand side of (2.6) is nonzero, whence $\operatorname{supp}(\mathbf{T}) \subseteq \operatorname{supp}(\mathbf{Q}) \oplus \mathcal{K}$. Let the $|b\rangle$ 's form an orthonormal basis in $\mathcal{K}$. Obviously, $\langle q \mid b\rangle=0$ for all $q$ and $b$. Then operator $\mathbf{T}$ can be represented as

$$
\mathbf{T}=\sum_{q} t_{q q}|q\rangle\left\langle q\left|+\sum_{q b}\left(t_{q b}|q\rangle\left\langle b\left|+t_{b q}\right| b\right\rangle\langle q|\right)+\sum_{b} t_{b b}\right| b\right\rangle\langle b| .
$$

As before, all the diagonal elements lie in the interval $[0 ; 1]$. Since the $\lambda_{q}$ 's in (1.6) are strictly positive, the condition $\operatorname{tr}_{1}\{\mathbf{T Q}\}=\operatorname{tr}_{1}(\mathbf{Q})$ implies that $t_{q q}=1$ for all values of label $q$. So the first sum in the right-hand side of (2.8) must be the projector onto $\operatorname{supp}(\mathbf{Q})$. Fixing some values of $q$ and $b$, we shall now consider the action of $\mathbf{T}$ in the two-dimensional subspace $\operatorname{span}\{|q\rangle,|b\rangle\}$. This action is described by the matrix

$$
\left(\begin{array}{cc}
1 & \gamma-i \delta \\
\gamma+i \delta & t_{b b}
\end{array}\right)
$$


Here $\gamma$ and $\delta$ are real, and $t_{q b}^{*}=t_{b q}=\gamma+i \delta$. By $\mathbf{T} \leq \mathbf{1}$ both eigenvalues of the matrix (2.9) are no greater than 1. This is valid if and only if $\gamma=\delta=0$ and therefore $t_{q b}=t_{b q}=0$. Let us denote the third sum in the right-hand side of $(2.8)$ by $\mathbf{M}$. It is obvious that this operator satisfies $\operatorname{supp}(\mathbf{M}) \subseteq \mathcal{K}$ and $\mathbf{0} \leq \mathbf{M} \leq \mathbf{1}$. Then the operator $\mathbf{T}$ is the sum of projector onto $\operatorname{supp}(\mathbf{Q})$ and $\mathbf{M}$.

The left-hand side of (2.4) can fruitfully be considered as a non-standard definition of the trace distance. The usual definition was seemingly inspired on the analogy of classicality (for details, see subsection 9.2.1 of reference [2]). In contrast, the series of arguments that leads to Theorem 1 is a self-contained nonclassical way to approach the genuine metric on quantum states. This way provides a kind of physical interpretation of equation (2.2) which is rather handy for evaluating the trace distance. Thus, we have arrived at the following definition.

Definition 2. (Non-standard definition of trace distance) The trace distance $D(\rho, \varrho)$ between quantum states $\rho$ and $\varrho$ is defined by

$$
D(\rho, \varrho):=\max _{\mathcal{E}}\left|\operatorname{tr}_{2}\{\mathcal{E}(\rho)\}-\operatorname{tr}_{2}\{\mathcal{E}(\varrho)\}\right| .
$$

The consistency of the new definition with the customary one is stated by Theorem 1. In connection with the definition given by (2.10) some unexpected questions are naturally risen. New insights into relationship of quantum operations and quantum states will be achieved by the study of these questions. Whenever the equality $d_{\mathcal{E}}(\rho, \varrho)=D(\rho, \varrho)$ is done by quantum operation $\mathcal{E}$, we will say: "the operation maximizes probability difference between $\rho$ and $\varrho "$. We ask: How many such quantum operations?

To each pair $\{\rho, \varrho\}$ of different states assign a family of classes labelled by integer $N>1$. The class specified by the given value $N$ contains an uncountably infinite number of those quantum operations that have $N$-dimensional output space and satisfy $d_{\mathcal{E}}(\rho, \varrho)=D(\rho, \varrho)$.

The claimed statement is justified as follows. Let us demand that operator $\mathbf{T}$ be equal to the projector onto $\operatorname{supp}(\mathbf{Q})$. We choose a relevant number of vectors $\left|q^{\prime}\right\rangle \in \mathcal{H}_{2}$ and take $\mathrm{E}_{q}=\left|q^{\prime}\right\rangle\langle q|$. The only thing we must assume about these vectors is that they are all normalized. In two and more dimensions, there are uncountably infinite number of ways to choose $\left|q^{\prime}\right\rangle$ 's. Thus, for any given value $N>1$ we can build an uncountably infinite number of those quantum operations that maximize probability difference between $\rho$ and $\varrho$, as claimed. The case, in which operator $\mathbf{T}$ should be equal to the projector 
onto $\operatorname{supp}(\mathbf{R})$, follows the same pattern. If $\operatorname{dim}(\mathcal{K})>0$ then by choice of $\mathbf{M}$ we obtain an additional freedom.

We have examined the question about those quantum operations that maximize probability difference between any prescribed two states. It is natural to inspect things in reverse order. As Theorem 1 shows, the specific property of considered quantum operations is that both the unity and zero are eigenvalues of $\mathbf{T}$. First, the operator $\mathbf{T}$ can be split into sum of projector and another operator with orthogonal supports. Second, the kernel of $\mathbf{T}$ is not zero-dimensional (except $\rho=\varrho$ ). So we pick out the special subclass of quantum operations. Let us begin with given quantum operation of described type. It is easy to build those two states that probability difference between them is maximized by the operation. In how many ways can we make such building?

A family of classes, labelled by real $\mathfrak{D} \in(0 ; 1)$, is assigned to each quantum operation $\mathcal{E}$ such that operator $\mathbf{T}$ has unit and zero eigenvalues. The class specified by the given value $\mathfrak{D}$ contains an uncountably infinite number of those pairs $\{\rho, \varrho\}$ that obey $D(\rho, \varrho)=d_{\mathcal{E}}(\rho, \varrho)=\mathfrak{D}$.

The justification is simple. We choose a nontrivial subspace of the eigenspace corresponding to unit eigenvalue of $\mathbf{T}$; this subspace is designed as $\operatorname{supp}(\mathbf{Q})$. Then we take a nontrivial subspace of the kernel of $\mathbf{T}$; that subspace is designed as $\operatorname{supp}(\mathbf{R})$. So, the conditions $\operatorname{tr}_{1}\{\mathbf{T Q}\}=\operatorname{tr}_{1}(\mathbf{Q})$ and $\operatorname{tr}_{1}\{\mathbf{T R}\}=0$ are provided. The orthogonal complement of $\operatorname{supp}(\mathbf{Q}) \oplus$ $\operatorname{supp}(\mathbf{R})$ is clearly designed as $\mathcal{K}$. Let the $|q\rangle$ 's and the $|r\rangle$ 's be those eigenvectors of $\mathbf{T}$ that form orthonormal sets in $\operatorname{supp}(\mathbf{Q})$ and $\operatorname{supp}(\mathbf{R})$ respectively. We then take positive numbers $\lambda_{q}$ and $\varkappa_{r}$ and define operators $\mathbf{Q}$ and $\mathbf{R}$ by (1.6) and (1.7), respectively. Both traces $\operatorname{tr}_{1}(\mathbf{Q})$ and $\operatorname{tr}_{1}(\mathbf{R})$ should be equal to $\mathfrak{D}$. That is, both the $\lambda_{q}$ 's and the $\varkappa_{r}$ 's sum to $\mathfrak{D}$. Then the trace distance between desired quantum states will be equal to $\mathfrak{D}$. We now aim to build normalized states $\rho$ and $\varrho$ satisfying $\rho-\varrho=\mathbf{Q}-\mathbf{R}$. We consider the case in which both $\rho$ and $\varrho$ are supported on $\operatorname{supp}(\mathbf{Q}) \oplus \operatorname{supp}(\mathbf{R})$ and diagonal with respect to the orthonormal set formed by $|q\rangle$ 's and $|r\rangle$ 's. Let us define these states as

$$
\begin{aligned}
\rho & :=\sum_{q}\left(\lambda_{q}+\delta \lambda_{q}\right)|q\rangle\left\langle q\left|+\sum_{r} \delta \varkappa_{r}\right| r\right\rangle\langle r|, \\
\varrho & :=\sum_{r}\left(\varkappa_{r}+\delta \varkappa_{r}\right)|r\rangle\left\langle r\left|+\sum_{q} \delta \lambda_{q}\right| q\right\rangle\langle q|,
\end{aligned}
$$


where positive variations $\delta \lambda_{q}$ and $\delta \varkappa_{r}$ must obey

$$
\sum_{q} \delta \lambda_{q}+\sum_{r} \delta \varkappa_{r}=1-\mathfrak{D}
$$

So the normalization of $\rho$ and $\varrho$ is provided. Because both sets $\left\{\delta \lambda_{q}\right\}$ and $\left\{\delta \varkappa_{r}\right\}$ are nonempty, we have an uncountably infinite number of ways to satisfy (2.13), as claimed above.

We have examined a maximum of $d_{\mathcal{E}}(\rho, \varrho)$ for the prescribed two states $\rho$ and $\varrho$. We shall now perform the maximization of $\mathcal{E}$-distance over all possible states. Consider a fixed quantum operation $\mathcal{E}$ of arbitrary type. It turns out that the desired maximum is equal to the difference between the maximal and minimal eigenvalues of operator T. By $\Theta$ and $\theta$ we respectively denote these maximal and minimal eigenvalues. Then the following statement holds.

Theorem 2. For arbitrary quantum operation $\mathcal{E}$,

$$
\max _{\rho, \varrho} d_{\mathcal{E}}(\rho, \varrho)=\Theta-\theta
$$

where the maximum is taken over all states $\rho$ and $\varrho$.

Proof. A value of $d_{\mathcal{E}}(\rho, \varrho)$ for particular two states $\rho$ and $\varrho$ is given by (2.5). In this equation two operators $\mathbf{Q}$ and $\mathbf{R}$ are uniquely determined by the two states. So both the trace of $\mathbf{Q}$ and the trace of $\mathbf{R}$ are equal to $D(\rho, \varrho)$. Under these conditions we can apply the result of Lemma 1 of Appendix A. By (A.1) the trace $\operatorname{tr}_{1}\{\mathbf{T Q}\}$ is no greater than $\Theta D(\rho, \varrho)$, by (A.2) the trace $\operatorname{tr}_{1}\{\mathbf{T R}\}$ is no less than $\theta D(\rho, \varrho)$. Therefore,

$$
d_{\mathcal{E}}(\rho, \varrho) \leq(\Theta-\theta) D(\rho, \varrho) \leq \Theta-\theta,
$$

where we used $D(\rho, \varrho) \leq 1$. The right-hand side of $(2.14)$ is reached under the following two conditions. The density operator $\rho$ must be multiplied by the normalizing factor projector onto nontrivial subspace of the eigenspace of $\mathbf{T}$ corresponding to eigenvalue $\Theta$; the density operator $\varrho$ must be multiplied by the normalizing factor projector onto nontrivial subspace of the eigenspace of $\mathbf{T}$ corresponding to eigenvalue $\theta$.

It is obvious that for trace-preserving quantum operation the $\mathcal{E}$-distance is equal to zero. In line with this fact, the right-hand side of (2.14) vanishes because $\mathbf{T}=\mathbf{1}$ for all trace-preserving operations. If quantum operation is maximizing, then both the unity and zero are eigenvalues of $\mathbf{T}$ and the righthand side of (2.14) is equal to 1 . The latter is the maximal acceptable value of $\mathcal{E}$-distance. This is another reason for usage of the word 'maximizing'. 


\section{Behaviour under the maximizing quantum operation}

In mutual relations of quantum operations and trace distance the following result of great moment is well known [2, 27]. Namely, no deterministic process increases the distance between two quantum states. That is, if $\mathcal{E}$ is a tracepreserving quantum operation then

$$
D(\mathcal{E}(\rho), \mathcal{E}(\varrho)) \leq D(\rho, \varrho)
$$

for arbitrary normalized states $\rho$ and $\varrho$. This result is usually referred to as contractivity of the trace distance under trace-preserving quantum operations. According to (1.5), for all trace-preserving operations $\mathbf{T}=\mathbf{1}$ and therefore states $\mathcal{E}(\rho)$ and $\mathcal{E}(\varrho)$ are normalized. The quantum operations that are the subject of interest in the present work do not preserve the trace. Nevertheless, the considered operations may be almost contractive in a specific sense. As has been shown above, with each quantum operation of the described type one can associate an uncountably infinite set of pairs with specified property. It is for these states that the following property of the operation is valid.

Theorem 3. If the quantum operation $\mathcal{E}$ maximizes probability difference between normalized states $\rho$ and $\varrho$ then

$$
D\left(\rho^{\prime}, \varrho^{\prime}\right) \leq p_{\mathrm{m}}^{-1} D(\rho, \varrho)
$$

where states $\rho^{\prime}$ and $\varrho^{\prime}$ are normalized outputs of the operation and $p_{\mathrm{m}}$ is maximum among two probabilities $\operatorname{tr}_{2}\{\mathcal{E}(\rho)\}$ and $\operatorname{tr}_{2}\{\mathcal{E}(\varrho)\}$.

Proof. We shall mean that $\rho \neq \varrho$ and therefore two probabilities are different. With no loss of generality, $\operatorname{tr}_{2}\{\mathcal{E}(\rho)\}>\operatorname{tr}_{2}\{\mathcal{E}(\varrho)\}$. This implies that the case (i) is realized (see the proof of Theorem 1). Due to (1.5) the

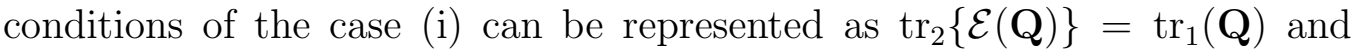
$\operatorname{tr}_{2}\{\mathcal{E}(\mathbf{R})\}=0$, whence

$$
\begin{aligned}
D(\rho, \varrho) & =\operatorname{tr}_{2}\{\mathcal{E}(\mathbf{Q})\}-\operatorname{tr}_{2}\{\mathcal{E}(\mathbf{R})\} \\
& \geq \operatorname{tr}_{2}\{\Pi \mathcal{E}(\mathbf{Q})\}-\operatorname{tr}_{2}\{\Pi \mathcal{E}(\mathbf{R})\} \\
& =\operatorname{tr}_{2}\{\Pi(\mathcal{E}(\rho)-\mathcal{E}(\varrho))\}
\end{aligned}
$$


for arbitrary projector $\Pi$. In the last line of (3.3) the linearity of the trace and the map (1.3) is used. According to (1.1) we further have

$$
\begin{aligned}
\rho^{\prime} & =p_{\mathrm{m}}^{-1} \mathcal{E}(\rho), \\
\varrho^{\prime} & =p_{\mathrm{n}}^{-1} \mathcal{E}(\varrho),
\end{aligned}
$$

where $p_{\mathrm{m}}=\operatorname{tr}_{2}\{\mathcal{E}(\rho)\}$ and $p_{\mathrm{n}}=\operatorname{tr}_{2}\{\mathcal{E}(\varrho)\}$. As it is well known (see equation (9.22) of reference [2]), there exists a projector $\Pi$ such that

$$
\operatorname{tr}_{2}\left\{\Pi\left(\rho^{\prime}-\varrho^{\prime}\right)\right\}=D\left(\rho^{\prime}, \varrho^{\prime}\right) .
$$

Using Eqs. (3.4) and (3.5) and inequality $p_{\mathrm{m}}>p_{\mathrm{n}}$ later, the last line of (3.3) can be put in the form

$$
p_{\mathrm{m}} \operatorname{tr}_{2}\left\{\Pi \rho^{\prime}\right\}-p_{\mathrm{n}} \operatorname{tr}_{2}\left\{\Pi \varrho^{\prime}\right\} \geq p_{\mathrm{m}} \operatorname{tr}_{2}\left\{\Pi\left(\rho^{\prime}-\varrho^{\prime}\right)\right\} \text {. }
$$

Combining this with (3.6) finally gives (3.2).

Thus, when the probability $p_{\mathrm{m}}$ is close to 1 , the value of $D\left(\rho^{\prime}, \varrho^{\prime}\right)$ is limited above by a quantity that is approximately equal to $D(\rho, \varrho)$. In this sense the considered operations may be related with trace-preserving quantum operations. For other values of $p_{\mathrm{m}}$ the upper bound given by (3.2) can appreciably exceed $D(\rho, \varrho)$. Nevertheless, this bound is nontrivial almost everywhere. Indeed, under the precondition of Theorem 3 we have $p_{\mathrm{m}}-p_{\mathrm{n}}=D(\rho, \varrho)$. So the right-hand side of (3.2) can be rewritten as $\left(1-p_{\mathrm{n}} / p_{\mathrm{m}}\right)$. If we represent $p_{\mathrm{m}}$ along the abscissa and $p_{\mathrm{n}}$ along the ordinate then the acceptable values of $p_{\mathrm{m}}$ and $p_{\mathrm{n}}$ lie in the rectangular triangle $0 \leq p_{\mathrm{n}}<p_{\mathrm{m}} \leq 1$. Except the side $p_{\mathrm{n}}=0$ of the triangle, the quantity $\left(1-p_{\mathrm{n}} / p_{\mathrm{m}}\right)$ is less than 1 and the bound given by (3.2) is therefore nontrivial.

To each point $\left(p_{\mathrm{m}}, p_{\mathrm{n}}\right)$ of the triangle assign normalized inputs $\rho$ and $\varrho$ such that $p_{\mathrm{m}}-p_{\mathrm{n}}=D(\rho, \varrho), p_{\mathrm{m}}=\operatorname{tr}_{2}\{\mathcal{E}(\rho)\}$ and $p_{\mathrm{n}}=\operatorname{tr}_{2}\{\mathcal{E}(\varrho)\}$ for given maximizing operation $\mathcal{E}$. Desired states are defined by (2.11) and (2.12), when both the $\lambda_{q}$ 's and the $\varkappa_{r}$ 's sum to $\left(p_{\mathrm{m}}-p_{\mathrm{n}}\right)$ and the right-hand side of (2.13) is equal to $\left(1-p_{\mathrm{m}}+p_{\mathrm{n}}\right)$. We shall now consider $D\left(\rho^{\prime}, \varrho^{\prime}\right)$ as a random variable with values from the interval $[0 ; 1]$. To evaluate average properties of a function of density matrices, it is necessary to define a certain measure in the set of considered ones [28]. In general, this is a subject of independent research. Some statistical properties of random density matrices have been analyzed by Sommers and Zyczkowski [29]. Problems of mentioned kind entail the specific tasks, such as computing the volume of set of mixed states 
with respect to the chosen measure [30, 31]. A discussion of these questions would take us to far afield.

Instead, we simply assume that all points of the triangle are equiprobable. Then the weight of those points that lead to $D\left(\rho^{\prime}, \varrho^{\prime}\right) \leq \xi$ is no less than $\xi$. Indeed, this inequality is provided by condition $p_{\mathrm{n}} \geq(1-\xi) p_{\mathrm{m}}$ together with (3.2). So the lower estimate $\xi$ is obtained as the ratio of areas of two triangles (the first triangle arises by section of the second one $0 \leq p_{\mathrm{n}}<p_{\mathrm{m}} \leq 1$ by line $\left.p_{\mathrm{n}}=(1-\xi) p_{\mathrm{m}}\right)$. In other words, the probability of event $D\left(\rho^{\prime}, \varrho^{\prime}\right) \leq \xi$ must be no less than $\xi$. The density equal to 1 corresponds to the probability distribution equal to $\xi$. By Lemma 2 of Appendix A, the $n$ 'th-order moment of $D\left(\rho^{\prime}, \varrho^{\prime}\right)$ is no greater than $1 /(n+1)$. In particular, the mean value does not exceed one half. We see that if the quantum operation maximizes the probability difference between inputs then the trace distance between outputs must take small values with significant frequency.

Unlike the trace-preserving quantum operations, the considered operations may increase the trace distance between two states. But if the probability difference between these states is maximized by given operation then a possible growth of the trace distance is limited above. In such a case the relative increase of the trace distance will be negligible by several times. Due to (3.2), a relative variation of the trace distance obeys

$$
\frac{D\left(\rho^{\prime}, \varrho^{\prime}\right)-D(\rho, \varrho)}{D\left(\rho^{\prime}, \varrho^{\prime}\right)} \leq 1-p_{\mathrm{m}} .
$$

We prove (3.7) for those pairs of states that satisfy the equality $d_{\mathcal{E}}=D$ for the given quantum operation $\mathcal{E}$. To any such pair we assign a point $\left(p_{\mathrm{m}}, p_{\mathrm{n}}\right)$ of the triangle $0 \leq p_{\mathrm{n}}<p_{\mathrm{m}} \leq 1$. Suppose those points in which the trace distance increases are uniformly distributed in the triangle. Estimate the weight of points such that the relative increase of trace distance is no greater than $\zeta$. This lower estimate is obtained as the ratio of the trapezoidal area severed by line $p_{\mathrm{m}}=1-\zeta$ from the triangle $0 \leq p_{\mathrm{n}}<p_{\mathrm{m}} \leq 1$ to the whole triangle area. We consider the relative increase of trace distance as a random variable with values from the interval $[0 ; 1]$. By calculations, the probability of the event that the random variable does not exceed $\zeta$ is no less than $\left(2 \zeta-\zeta^{2}\right)$. The latter distribution is assigned to the density equal to $(2-2 \zeta)$. Due to Lemma 2, the $n$ 'th-order moment of the random variable does not exceed $2 /\left(n^{2}+3 n+2\right)$. In particular, the mean value is less than or equal to one third. Thus, on the average the relative increase of trace distance is 
not great. Such a property seems to be similar to the contractivity under trace-preserving quantum operations.

There is another characterization of behaviour of the trace distance under quantum operations maximizing probability difference between their inputs. In some instances, the formulation in terms of subnormalized outputs may be more embossed than (3.2). Except the trace-preserving operations, the output $\mathcal{E}(\rho)$ is subnormalized, i.e. $\operatorname{tr}_{2}\{\mathcal{E}(\rho)\} \leq 1$. So an extension of the notion of trace distance to subnormalized states is needed. A study of the general case is beyond the scope of this paper. However, we can give a transparent outline of the case of Hermitian operators. All the necessary details are gathered in Appendix B. It is proved there that the trace distance is a metric on the space of Hermitian operators. We can now establish the desired characterization.

Theorem 4. If the quantum operation $\mathcal{E}$ maximizes probability difference between normalized states $\rho$ and $\varrho$ then

$$
D(\mathcal{E}(\rho), \mathcal{E}(\varrho)) \leq \frac{1}{2} D(\rho, \varrho)
$$

Proof. We again suppose that $\operatorname{tr}_{2}\{\mathcal{E}(\rho)\}>\operatorname{tr}_{2}\{\mathcal{E}(\varrho)\}$. Due to the precondition of Theorem 4 , the difference between these traces is equal to $D(\rho, \varrho)$. Using this fact and (B.3), we see that there exists a projector $\Pi$ such that

$$
\operatorname{tr}_{2}\{\Pi(\mathcal{E}(\rho)-\mathcal{E}(\varrho))\}=D(\mathcal{E}(\rho), \mathcal{E}(\varrho))+\frac{1}{2} D(\rho, \varrho)
$$

Combining this with (3.3), after cancellation we obtain (3.8).

Like (3.2), in Theorem 4 the nontrivial upper bound on the trace distance between outputs is established. Namely, if the quantum operation maximizes probability difference between inputs then the trace distance between outputs is at most one-half of the trace distance between inputs. Assume that all the points of the triangle $0 \leq p_{\mathrm{n}}<p_{\mathrm{m}} \leq 1$ are equiprobable. Then the mean value of $D(\rho, \varrho)$ is equal to one third. This result is obtained as the ratio of the integral of $\left(p_{\mathrm{m}}-p_{\mathrm{n}}\right)$ over triangle to the area of triangle. By (3.8), the mean value of $D(\mathcal{E}(\rho), \mathcal{E}(\varrho))$ is no greater than one sixth. Thus, on the average the outputs must be enough close.

We see from (3.8) that for examined operations the trace distance between subnormalized outputs is bounded above when the inputs form a pair from specified class. At the same time, there is an important example of opposite behaviour of the trace distance. Let us consider a procedure of approximate 
(or probabilistic) duplicating quantum states called 'quantum cloning' and useful in many tasks of quantum information processing. Concrete limitations of this procedure follow from its specification [32]. After inspiring paper by Bužek and Hillery [33], the much various scenarios have been studied the deterministic cloning [34, 35, 36, 37] and the probabilistic cloning [38, 39], the hybrid scheme [40, 41], the cloning with prior information [42, 43, 44], applications to joint measurement of noncommuting observables [45, 46], and the tasks [47, 48, 49] connected with the quantum cryptography.

Exact clones may be generated by probabilistic process only. Optimal exact cloning of state secretly chosen from a certain pair of different pure states $\omega_{1}$ and $\omega_{2}$ has the success probability $1 /(1+\Omega)$, where $\Omega$ denotes the fidelity of (normalized) states $\omega_{1}$ and $\omega_{2}$ [38, 39]. Recall that the fidelity of normalized states $\rho$ and $\varrho$ is defined by [2, 50]

$$
F(\rho, \varrho):=\operatorname{tr}_{1} \sqrt{\sqrt{\rho} \varrho \sqrt{\rho}} .
$$

[Such a usage of the word 'fidelity' is not unique. In [51] Jozsa introduced this word for Uhlmann's transition probability [52] equal to square of the right-hand side of (3.9).] For normalized states the trace distance and the fidelity are related by the inequality

$$
D(\rho, \varrho) \leq \sqrt{1-F^{2}(\rho, \varrho)}
$$

which is always saturated for pure states [2]. The actual outputs of exact cloning operation $\mathcal{G}$ is expressed as

$$
\mathcal{G}\left(\omega_{j}\right)=(1+\Omega)^{-1} \omega_{j} \otimes \omega_{j}
$$

where $j=1,2$. By multiplicativity of the fidelity [51], we have $F\left(\omega_{1} \otimes \omega_{1}, \omega_{2} \otimes\right.$ $\left.\omega_{2}\right)=F^{2}\left(\omega_{1}, \omega_{2}\right)=\Omega^{2}$. Since both the states $\omega_{j}$ and $\omega_{j} \otimes \omega_{j}$ are pure, the equality in (3.10) holds whence

$$
D\left(\omega_{1} \otimes \omega_{1}, \omega_{2} \otimes \omega_{2}\right)=\sqrt{1+\Omega^{2}} D\left(\omega_{1}, \omega_{2}\right) .
$$

Using the customary definition of the trace distance, equation (3.11) and the last relation, we then obtain

$$
D\left(\mathcal{G}\left(\omega_{1}\right), \mathcal{G}\left(\omega_{2}\right)\right)=\frac{\sqrt{1+\Omega^{2}}}{1+\Omega} D\left(\omega_{1}, \omega_{2}\right)
$$


Because the normalized states $\omega_{1}$ and $\omega_{2}$ are different, a value of $\Omega=$ $F\left(\omega_{1}, \omega_{2}\right)$ lies in the interval $[0 ; 1)$. For such values the multiplier of $D\left(\omega_{1}, \omega_{2}\right)$ in (3.12) is decreasing function of $\Omega$ and, therefore, is greater than $1 / \sqrt{2}$. Thus, if the quantum operation $\mathcal{G}$ is designed to clone exactly the prescribed pure states $\omega_{1}$ and $\omega_{2}$ then

$$
D\left(\mathcal{G}\left(\omega_{1}\right), \mathcal{G}\left(\omega_{2}\right)\right)>\frac{1}{\sqrt{2}} D\left(\omega_{1}, \omega_{2}\right) .
$$

Let us compare the two results established by equations (3.8) and (3.13) respectively. The similarity is that each of these results imposes some bound on the trace distance between two outputs when the two input states form specified pair. The differences are significant in the following respects. First, the trace distance between outputs of considered operation $\mathcal{E}$ is bounded above, the trace distance between outputs of exact cloning operation $\mathcal{G}$ is bounded below. Second, inequality (3.8) is valid for infinitely many pairs of inputs, inequality (3.13) is valid for only one pair of inputs. The more demonstrative of the two differences is the first. The second difference is rather a manifestation of the fact that in physical processes a loss of distinguishability usually occurs.

\section{Relations with sine distance}

In this section, we shall discuss a relationship of the trace distance and a close measure that is called 'sine distance' in [26. There are the two useful definitions of the sine distance. The first definition is based on the concept of purifications and the notion of angle between quantum states. In [53] the angle $\Delta(\rho, \varrho) \in[0 ; \pi / 2]$ between states $\rho$ and $\varrho$ has been defined by

$$
\Delta(\rho, \varrho):=\min _{|\Phi\rangle,|\Psi\rangle} \Delta(|\Phi\rangle,|\Psi\rangle)
$$

where the minimization is over all purifications $|\Phi\rangle$ of $\rho$ and $|\Psi\rangle$ of $\varrho$, and $\Delta(|\Phi\rangle,|\Psi\rangle):=\arccos |\langle\Phi \mid \Psi\rangle|$. The sine distance between states $\rho$ and $\varrho$ is then defined as [26]

$$
C(\rho, \varrho):=\sin \Delta(\rho, \varrho) .
$$

The name 'sine distance' has been arisen from (4.1). According to the second definition [26], the sine distance $C(\rho, \varrho)$ is defined as the right-hand side of 
(3.10). These definitions are consistent, because there holds [2]

$$
F(\rho, \varrho)=\cos \Delta(\rho, \varrho) .
$$

It turned out that the sine distance is useful in the state-dependent quantum cloning. Following [34], state-dependent cloners are usually evaluated with respect to those figures of merit that are based on the fidelity. In [43, 54] the new figure of merit, based on the sine distance and called 'relative error', has been proposed. A study of cloners with respect to the relative error has allowed us to complete the portrait of state-dependent cloning [54]. In addition, the considered distance seems to be useful in the context of quantum computation [55].

If both the states are pure, the equality in (3.10) takes place and, therefore, the sine distance is equal to the trace distance. In general, however, the sine distance can be larger than the trace distance. Consider the pure state $|0\rangle\langle 0|$ and the mixed state $\varrho$ with spectral decomposition

$$
\varrho=(1-\lambda)|0\rangle\left\langle 0\left|+\sum_{r \neq 0} \varkappa_{r}\right| r\right\rangle\langle r| .
$$

It is easy to check that $F(|0\rangle, \varrho)=\sqrt{1-\lambda}$, whence $C(|0\rangle, \varrho)=\sqrt{\lambda}$. Splitting operator $(|0\rangle\langle 0|-\varrho)$ into positive and negative parts is obvious, and from (2.3) we obtain $D(|0\rangle, \varrho)=\lambda$. The maximum of function $\sqrt{\lambda}-\lambda=1 / 4-$ $(\sqrt{\lambda}-1 / 2)^{2}$ is equal to one forth and reached at $\lambda=1 / 4$. So for this value of $\lambda$ we have

$$
C(|0\rangle, \varrho)-D(|0\rangle, \varrho)=1 / 4 .
$$

Theorem 5. The maximum of difference between the sine distance and the trace distance satisfies

$$
\frac{1}{4} \leq \max _{\rho, \varrho}\{C(\rho, \varrho)-D(\rho, \varrho)\} \leq \sqrt{2}-1,
$$

where the maximization is over all states $\rho$ and $\varrho$.

Proof. The lower bound follows from (4.3). As is shown in [2, 56, $1-F(\rho, \varrho) \leq D(\rho, \varrho)$ whence

$$
C(\rho, \varrho)-D(\rho, \varrho) \leq C(\rho, \varrho)+F(\rho, \varrho)-1 .
$$

Due to (4.1) and (4.2), the last inequality can be rewritten as

$$
C(\rho, \varrho)-D(\rho, \varrho) \leq \sin \Delta(\rho, \varrho)+\cos \Delta(\rho, \varrho)-1 .
$$


The upper bound is provided by (4.5) and Lemma 3 of Appendix A.

It is not insignificant that in the case of single qubits the lower bound in (4.4) is saturated. In other words, the maximum of difference between the sine distance and the trace distance is equal to one forth. As always, we represent the density matrices by $\rho=(1 / 2)\{\mathbf{1}+\vec{u} \cdot \vec{\sigma}\}$ and $\varrho=(1 / 2)\{\mathbf{1}+\vec{v} \cdot \vec{\sigma}\}$. Here $\vec{u}$ and $\vec{v}$ are Bloch vectors and $\vec{\sigma}$ denotes the three-component vector of Pauli matrices. The square of the fidelity of states $\rho$ and $\varrho$ is then expressed as 51

$$
F^{2}(\rho, \varrho)=\frac{1}{2}\left\{1+\vec{u} \cdot \vec{v}+\sqrt{1-u^{2}} \sqrt{1-v^{2}}\right\} .
$$

Next, the trace distance between two single qubit states is equal to one-half of modulus of difference between their Bloch vectors [2]. So the difference between the sine distance and the trace distance is equal to the function

$$
\begin{aligned}
f(u, v, \eta)= & \frac{1}{\sqrt{2}}\left\{1-u v \eta-\sqrt{1-u^{2}} \sqrt{1-v^{2}}\right\}^{1 / 2} \\
& -\frac{1}{2}\left\{u^{2}+v^{2}-2 u v \eta\right\}^{1 / 2},
\end{aligned}
$$

where $\eta$ denotes the cosine of angle between $\vec{u}$ and $\vec{v}$. Acceptable values of variables $u, v$ and $\eta$ lie in the parallelepiped defined by $0 \leq u \leq 1$, $0 \leq v \leq 1$ and $-1 \leq \eta \leq 1$. Finding maximum of the function $f(u, v, \eta)$ in the parallelepiped is a task of elementary calculus. It has been verified that desired maximum is equal to one forth. But we refrain from presenting the calculations here.

To sum up we see that the trace distance is closely related to the sine distance. Moreover, in the case of pure states the two distance measures are equal to each other. In general, the sine distance can be larger than the trace distance. So the trace distance is sometimes tighter. But the maximum of difference between the sine distance and the trace distance lies between values $1 / 4$ and $(\sqrt{2}-1)$. The former takes place in the case of single qubits. It would be interesting to study a dependence of this maximum on the dimensionality of state space. But this problem seems to be enough difficult.

\section{Conclusion}

We have considered the trace distance from the viewpoint of quantum operation formalism. The new definition of trace distance in terms of a maximum 
over all quantum operations was proposed. The definition proposed in this paper has the advantage of a physical interpretation of the trace distance in terms of quantum operations. In connection with this definition the interesting subclass of maximizing quantum operation was specified. It has been shown that each of such operations maximizes a difference between two probabilities generated by the operation on some pairs of inputs. For each pair of different states there exist an uncountably infinite number of quantum operations with specified property. Conversely, for each quantum operation of described type there exist an uncountably infinite number of pairs of those states that probability difference between them is maximized by the operation.

It turned out that if quantum operation maximizes the probability difference between inputs then the trace distance between outputs is bounded above. Due to made estimates of trace distance between outputs, described operations have been related to the trace-preserving quantum operations. The revealed property seems to be similar to the well-known contractivity under the trace-preserving quantum operations. But this property is valid only for specific pairs of inputs. Finally, we have discussed relations of the trace distance to a measure called 'sine distance'. The lower and upper bounds on the maximum of difference between the sine distance and the trace distance were obtained. In the case of single qubits the exact value of this maximum is mentioned. These results show that the sine distance and the trace distance are closely related. 


\section{A Three lemmas}

Let us consider a product of two positive operators, one of which is fixed and other of which is freely variable. We find the maximal and minimal values of the trace of this product. By $\Theta$ and $\theta$, we denote the maximal and minimal eigenvalues of the fixed positive operator $\mathbf{T}$, respectively. Then the following statement takes place.

Lemma 1. For the given positive operator $\mathbf{T}$,

$$
\begin{aligned}
& \max _{\operatorname{tr}(\mathbf{Q})=\mathfrak{D}} \operatorname{tr}(\mathbf{T} \mathbf{Q})=\Theta \cdot \mathfrak{D}, \\
& \min _{\operatorname{tr}(\mathbf{Q})=\mathfrak{D}} \operatorname{tr}(\mathbf{T} \mathbf{Q})=\theta \cdot \mathfrak{D},
\end{aligned}
$$

where both the maximization and minimization is over all positive operators $\mathbf{Q}$ satisfying $\operatorname{tr}(\mathbf{Q})=\mathfrak{D}$.

Proof. Using the spectral decomposition of operator $\mathbf{T}$ and the definitions of $\theta$ and $\Theta$, we obtain that for each normalized state $|q\rangle$

$$
\theta \leq\langle q|\mathbf{T}| q\rangle \leq \Theta
$$

Due to the properties of the trace and (1.6), $\operatorname{tr}(\mathbf{T Q})=\sum_{q} \lambda_{q}\langle q|\mathbf{T}| q\rangle$. This, when combined with (A.3), finally gives

$$
\theta \cdot \mathfrak{D} \leq \operatorname{tr}(\mathbf{T Q}) \leq \Theta \cdot \mathfrak{D}
$$

Here we used that $\operatorname{tr}(\mathbf{Q})=\sum_{q} \lambda_{q}=\mathfrak{D}$. To reach the lower bound in (A.4) we take a nontrivial subspace of the eigenspace of $\mathbf{T}$ corresponding to eigenvalue $\theta$; then $\mathbf{Q}$ should be the projector onto this subspace multiplied by the ratio of $\mathfrak{D}$ to trace of the projector. To reach the upper bound in (A.4) we take a nontrivial subspace of the eigenspace of $\mathbf{T}$ corresponding to eigenvalue $\Theta$; then $\mathrm{Q}$ should be the projector onto that subspace multiplied by the ratio of $\mathfrak{D}$ to trace of the projector.

Let $X$ and $Y$ be the real-valued random variables with probability densities $g(x)$ and $h(y)$ respectively. It is sufficient for our aims to consider only those probability densities that vanish outside a certain interval $[0 ; R]$. A distribution function of $\xi$ is defined as the probability that a value of the random variable is no greater than $\xi$ [57]. This function is obtained by integration from 0 to $\xi$ of corresponding probability density. The moments are 
important quantitative indices of distribution properties [57]. In our case the $n$ 'th-order moments of $X$ and $Y$ are expressed by

$$
\begin{aligned}
\left\langle X^{n}\right\rangle & =\int_{0}^{R} x^{n} g(x) d x, \\
\left\langle Y^{n}\right\rangle & =\int_{0}^{R} y^{n} h(y) d y .
\end{aligned}
$$

We shall now show that if the two distribution functions satisfy the same inequality for all $\xi$ in $[0 ; R]$, then the two moments of $n$ 'th order satisfy the opposite inequality.

Lemma 2. If there holds $\int_{0}^{\xi} g(x) d x \geq \int_{0}^{\xi} h(y) d y$ for all $\xi \in[0 ; R]$ then

$$
\left\langle X^{n}\right\rangle \leq\left\langle Y^{n}\right\rangle \quad(n>0) .
$$

Proof. The quantity $n y^{n-1} \int_{0}^{y}\{g(x)-h(x)\} d x$ is nonnegative for all $y \in[0 ; R]$ due to the precondition of Lemma 2. So by integration from $y=0$ to $y=R$ of this nonnegative quantity we obtain

$$
\begin{aligned}
& \int_{0}^{R} d y \int_{0}^{y} d x n y^{n-1}\{g(x)-h(x)\} \\
& =\int_{0}^{R} d x \int_{x}^{R} d y n y^{n-1}\{g(x)-h(x)\} \\
& =\int_{0}^{R} d x\left(R^{n}-x^{n}\right)\{g(x)-h(x)\} \geq 0 .
\end{aligned}
$$

In the last line of (A.8) the multiplier of $R^{n}$ is zero by the normalization of densities. Combining this with (A.5) and (A.6) finally gives (A.7).

It should be noted that the above result remains valid when the probability densities are distributed among the whole positive semiaxis. To prove this we must consider the limit $R \rightarrow+\infty$. It turns out that if the integrals in (A.5) and (A.6) are convergent then the statement of Lemma 2 is still correct. We do not enter into details here because in Section 3 we deal with probability densities concentrated on the interval [0;1]. In general, Lemma 2 can be extended to any function of the random variable such that its derivative is nonnegative in those intervals on which the densities are concentrated. A discussion of this question would be out of the place here.

Lemma 3. For arbitrary angle $\alpha$ there holds

$$
\sin \alpha+\cos \alpha \leq \sqrt{2} \text {. }
$$


Proof. By doing usual trigonometry, we obtain

$$
\begin{aligned}
\sin \alpha+\cos \alpha & =\sqrt{2}(\sin \alpha \cos (\pi / 4)+\cos \alpha \sin (\pi / 4)) \\
& =\sqrt{2} \sin (\alpha+\pi / 4) .
\end{aligned}
$$

Because the sine does not exceed one, this equality provides (A.9).

\section{B Trace distance between Hermitian operators}

In general, the right-hand side of (2.2) can naturally be extended in much broad context. Indeed, the expression for trace distance between two density operators is regardless of the normalization and the positivity of them. We shall restrict our consideration to the case of Hermitian operators. In the first place, this subclass of operators is extremely important. In the second

place, under such a restriction we can give a simple analysis of the properties of the trace distance. The trace distance between Hermitian operators A and $B$ is defined by

$$
D(\mathrm{~A}, \mathrm{~B}):=\frac{1}{2} \operatorname{tr}|\mathrm{A}-\mathrm{B}| .
$$

Due to Hermiticity of A and B we can obtain a direct analogue of (2.3). Like a difference between density matrices, Hermitian operator $(A-B)$ can be written as $\mathbf{A}-\mathbf{B}=\mathbf{P}-\mathbf{S}$, where $\mathbf{P}$ and $\mathbf{S}$ are positive operators with orthogonal supports. These operators are got from the spectral decomposition of $(A-B)$ by the same way that leads to (1.6) and (1.7). Drawing analogy with (2.3), we immediately obtain

$$
D(\mathrm{~A}, \mathrm{~B})=\frac{1}{2} \operatorname{tr}(\mathbf{P})+\frac{1}{2} \operatorname{tr}(\mathbf{S}) .
$$

In contrast to the case of normalized density operators, neither $\operatorname{tr}(\mathbf{P})$ nor $\operatorname{tr}(\mathbf{S})$ are equal to the right-hand side of $(\overline{B .2})$ (except when $\operatorname{tr}(\mathrm{A})=\operatorname{tr}(\mathrm{B})$ solely).

The distance defined by (B.1) is just a metric on the space of Hermitian operators. It is obvious that the distance takes nonnegative real values, that $D(\mathrm{~A}, \mathrm{~B})=0$ if and only if $\mathrm{A}=\mathrm{B}$, and that $D(\mathrm{~A}, \mathrm{~B})=D(\mathrm{~B}, \mathrm{~A})$. The only vague step is a proof of the triangle inequality. Here a generalization of (3.6) is needed. 
Lemma 4. For arbitrary two Hermitian operators A and B

$$
\max _{\Pi \leq 1} \operatorname{tr}\{\Pi(\mathrm{A}-\mathrm{B})\}=D(\mathrm{~A}, \mathrm{~B})+\frac{\operatorname{tr}(\mathrm{A})-\operatorname{tr}(\mathrm{B})}{2},
$$

where maximum is taken over all positive operators $\Pi$ satisfying $\Pi \leq \mathbf{1}$ (or alternately over all projectors).

Proof. Taking the trace of operator $A-B=\mathbf{P}-\mathbf{S}$ and using $(\overline{B .2})$, we obtain

$$
D(\mathrm{~A}, \mathrm{~B})+\frac{1}{2}[\operatorname{tr}(\mathrm{A})-\operatorname{tr}(\mathrm{B})]=\operatorname{tr}(\mathbf{P}) .
$$

Prove that the left-hand side of $(\underline{\mathrm{B} .3})$ is equal to $\operatorname{tr}(\mathbf{P})$. For any positive operator $\Pi \leq \mathbf{1}$ there holds

$$
\operatorname{tr}\{\Pi(A-B)\}=\operatorname{tr}\{\Pi(\mathbf{P}-\mathbf{S})\} \leq \operatorname{tr}\{\Pi \mathbf{P}\} \leq \operatorname{tr}(\mathbf{P}) .
$$

When $\Pi$ is the projector onto the support of $\mathbf{P}$, both the last inequalities are saturated.

Note that Lemma 4 is related in kinship to Theorem 1. In (B.3) the maximization is over all positive operators $\Pi$ meeting $\Pi \leq \mathbf{1}$. If we substitute the defined by (1.4) operator $\mathbf{T}$ for abstract $\Pi$ then in the left-hand side of (B.3) we obtain the maximum over all quantum operations. In this sense, the statement of Theorem 1 provides a kind of physical interpretation of (B.3) for the case of density operators. Besides, in Theorem 1 the explicit conditions of achievement of the maximum are established. On other hand, Lemma 4 deals with arbitrary Hermitian operators. Furthermore, its applications to the proof of the triangle inequality and the convexity do not involve conditions of maximum achievement. We now note from (B.3) that there exists a projector $\Pi$ such that

$$
\operatorname{tr}\{\Pi(\mathrm{A}-\mathrm{B})\}-\frac{1}{2}[\operatorname{tr}(\mathrm{A})-\operatorname{tr}(\mathrm{B})]=D(\mathrm{~A}, \mathrm{~B}) .
$$

In accordance with Lemma 4, we further have

$$
\begin{aligned}
& \operatorname{tr}\{\Pi(\mathrm{A}-\mathrm{C})\}-\frac{1}{2}[\operatorname{tr}(\mathrm{A})-\operatorname{tr}(\mathrm{C})] \leq D(\mathrm{~A}, \mathrm{C}), \\
& \operatorname{tr}\{\Pi(\mathrm{C}-\mathrm{B})\}-\frac{1}{2}[\operatorname{tr}(\mathrm{C})-\operatorname{tr}(\mathrm{B})] \leq D(\mathrm{C}, \mathrm{B}) .
\end{aligned}
$$

Summing the two last inequalities and using (B.4), we finally obtain that $D(\mathrm{~A}, \mathrm{~B}) \leq D(\mathrm{~A}, \mathrm{C})+D(\mathrm{C}, \mathrm{B})$. Thus, the triangle inequality holds too. 
The trace distance between density matrices satisfies the following two properties: the joint convexity and the convexity [2]. These properties remain valid for Hermitian matrices. Let $\left\{p_{j}\right\}$ be probability distribution, and $\mathrm{A}_{j}$ and $\mathrm{B}_{j}$ be Hermitian operators with labels from the same set. Then

$$
D\left(\sum_{j} p_{j} \mathrm{~A}_{j}, \sum_{j} p_{j} \mathrm{~B}_{j}\right) \leq \sum_{j} p_{j} D\left(\mathrm{~A}_{j}, \mathrm{~B}_{j}\right),
$$

that is the trace distance is jointly convex in its inputs. Substituting $C$ for all $\mathrm{B}_{j}$ 's into (B.5) and using the condition $\sum_{j} p_{j}=1$, we obtain

$$
D\left(\sum_{j} p_{j} \mathrm{~A}_{j}, \mathrm{C}\right) \leq \sum_{j} p_{j} D\left(\mathrm{~A}_{j}, \mathrm{C}\right) .
$$

That is, the trace distance is convex function on the set of Hermitian matrices.

The proof of (B.5) is simple. By A and B we denote $\sum_{j} p_{j} \mathrm{~A}_{j}$ and $\sum_{j} p_{j} \mathrm{~B}_{j}$ respectively. Due to (B.3) there exists a projector $\Pi$ such that

$$
\begin{aligned}
D(\mathrm{~A}, \mathrm{~B}) & =\operatorname{tr}\{\Pi(\mathrm{A}-\mathrm{B})\}-\frac{1}{2}[\operatorname{tr}(\mathrm{A})-\operatorname{tr}(\mathrm{B})] \\
& =\sum_{j} p_{j} \operatorname{tr}\left\{\Pi\left(\mathrm{A}_{j}-\mathrm{B}_{j}\right)\right\}-\frac{1}{2}[\operatorname{tr}(\mathrm{A})-\operatorname{tr}(\mathrm{B})] \\
& \leq \sum_{j} p_{j}\left\{D\left(\mathrm{~A}_{j}, \mathrm{~B}_{j}\right)+\frac{1}{2}\left[\operatorname{tr}\left(\mathrm{A}_{j}\right)-\operatorname{tr}\left(\mathrm{B}_{j}\right)\right]\right\} \\
& -\frac{1}{2}[\operatorname{tr}(\mathrm{A})-\operatorname{tr}(\mathrm{B})] .
\end{aligned}
$$

Here in the last part of (B.6) the statement of Lemma 4 was applied. After cancellation in this part we obtain (B.5).

\section{References}

[1] Kraus K 1983 States, Effects and Operations: Fundamental Notions of Quantum Theory (Lecture Notes in Physics vol. 190) (Berlin: SpringerVerlag)

[2] Nielsen M A and Chuang I L 2000 Quantum Computation and Quantum Information (Cambridge: Cambridge University Press)

[3] Hellwig K-E and Kraus K 1969 Commun. Math. Phys. 11214 
[4] Hellwig K-E and Kraus K 1970 Commun. Math. Phys. 16142

[5] Kraus K 1971 Ann. Phys. 64311

[6] Lindblad G 1975 Commun. Math. Phys. 40147

[7] Choi M-D 1975 Linear Algebra Appl. 10285

[8] Bennett C H 1992 Phys. Rev. Lett. 683121

[9] Olivares S and Paris M G A 2004 J. Opt. B: Quantum Semiclass. Opt. 669

[10] Helstrom C W 1976 Quantum Detection and Estimation Theory (New York: Academic Press)

[11] Ivanovic I D 1987 Phys. Lett. A 123257

[12] Dieks D 1988 Phys. Lett. A 126303

[13] Peres A 1988 Phys. Lett. A 12819

[14] Chefles A 1998 Phys. Lett. A 239339

[15] Chefles A and Barnett S M 1998 Phys. Lett. A 250223

[16] Rudolph T, Spekkens R W and Turner P S 2003 Phys. Rev. A 68 010301(R)

[17] Peres A and Terno D R 1998 J. Phys. A: Math. Gen. 347105

[18] Barnum H, Caves C M, Fuchs C A, Jozsa R and Schumacher B 1996 Phys. Rev. Lett. 762818

[19] Nielsen M A and Caves C M 1997 Phys. Rev. A 552547

[20] Chefles A and Barnett S M 1998 J. Phys. A: Math. Gen. 3110097

[21] Qiu D 2002 J. Phys. A: Math. Gen. 356931

[22] Feng Y, Duan R and Ji Z 2005 Phys. Rev. A 72012313

[23] Fiurašek J and Ježek M 2003 Phys. Rev. A 67012321 
[24] Aharonov D, Kitaev A and Nisan N 1998 Quantum circuits with mixed states Preprint quant-ph/9806029

[25] Reed M and Simon B 1972 Methods of Modern Mathematical Physics vol. 1 Functional Analysis (New York: Academic Press)

[26] Rastegin A E 2006 Sine distance for quantum states Preprint quant-ph/0602112

[27] Ruskai M B 1994 Rev. Math. Phys. 61147

[28] Zyczkowski K and Sommers H-J 2001 J. Phys. A: Math. Gen. 347111

[29] Sommers H-J and Zyczkowski K 2004 J. Phys. A: Math. Gen. 378457

[30] Sommers H-J and Zyczkowski K 2003 J. Phys. A: Math. Gen. 3610083

[31] Zyczkowski K and Sommers H-J 2003 J. Phys. A: Math. Gen. 3610115

[32] Scarani V, Iblisdir S, Gisin N and Acin A 2005 Rev. Mod. Phys. 771225

[33] Bužek V and Hillery M 1996 Phys. Rev. A 541844

[34] Bruß D, DiVincenzo D P, Ekert A, Fuchs C A, Macchiavello C and Smolin J A 1998 Phys. Rev. A 572368

[35] Werner R F 1998 Phys. Rev. A 581827

[36] Keyl M and Werner R F 1999 J. Math. Phys. 403283

[37] Macchiavello C 2000 J. Opt. B: Quantum Semiclass. Opt. 2144

[38] Duan L-M and Guo G-C 1998 Phys. Rev. Lett. 804999

[39] Duan L-M and Guo G-C 1998 Phys. Lett. A 243261

[40] Chefles A and Barnett S M 1999 Phys. Rev. A 60136

[41] Fiurašek J 2004 Phys. Rev. A 70032308

[42] Rastegin A E 2003 Phys. Rev. A 68032303

[43] Rastegin A E 2003 J. Opt. B: Quantum Semiclass. Opt. 5 S647 
[44] Qiu D 2006 J. Phys. A: Math. Gen. 395135

[45] D'Ariano G M, Macchiavello C and Sacchi M F 2001 J. Opt. B: Quantum Semiclass. Opt. 344

[46] Brougham T, Andersson E and Barnett S M 2006 Phys. Rev. A 73 062319

[47] Niu C-S and Griffiths R B 1999 Phys. Rev. A 602764

[48] Bruß D and Macchiavello C 2001 J. Phys. A: Math. Gen. 346815

[49] Cerf N J, Bourennane M, Karlsson A and Gisin N 2002 Phys. Rev. Lett. 88127902

[50] Uhlmann A 2000 Rep. Math. Phys. 45407

[51] Jozsa R 1994 J. Mod. Opt. 412315

[52] Uhlmann A 1976 Rep. Math. Phys. 9273

[53] Rastegin A E 2003 Phys. Rev. A 67012305

[54] Rastegin A E 2002 Phys. Rev. A 66042304

[55] Gilchrist A, Langford N K and Nielsen M A 2005 Phys. Rev. A 71 062310

[56] Fuchs C A and van de Graaf J 1999 IEEE Trans. Inf. Theory 451216

[57] Feller W 1971 An Introduction to Probability Theory and its Applications vol. II (New York: Wiley) 